\title{
Automatic segmentation and classification of breast lesions through identification of informative multiparametric PET/MRI features
}

Wolf-Dieter Vogl ${ }^{1 \dagger}$, Katja Pinker ${ }^{2,3 \dagger}$, Thomas H. Helbich², Hubert Bickel ${ }^{2}$, Günther Grabner ${ }^{4,7}$, Wolfgang Bogner ${ }^{4}$, Stephan Gruber ${ }^{4}, Z^{2}$ suzsanna Bago-Horvath ${ }^{5}$, Peter Dubsky ${ }^{6}$ and Georg Langs ${ }^{1 *}$

\begin{abstract}
Background: Multiparametric positron emission tomography/magnetic resonance imaging (mPPET/MRI) shows clinical potential for detection and classification of breast lesions. Yet, the contribution of features for computeraided segmentation and diagnosis (CAD) need to be better understood. We proposed a data-driven machine learning approach for a CAD system combining dynamic contrast-enhanced (DCE)-MRl, diffusion-weighted imaging (DWI), and ${ }^{18} \mathrm{~F}$-fluorodeoxyglucose $\left({ }^{18} \mathrm{~F}\right.$-FDG)-PET.

Methods: The CAD incorporated a random forest (RF) classifier combined with mpPET/MRI intensity-based features for lesion segmentation and shape features, kinetic and spatio-temporal texture features, for lesion classification. The CAD pipeline detected and segmented suspicious regions and classified lesions as benign or malignant. The inherent feature selection method of RF and alternatively the minimum-redundancy-maximum-relevance feature ranking method were used.

Results: In 34 patients, we report a detection rate of 10/12 (83.3\%) and 22/22 (100\%) for benign and malignant lesions, respectively, a Dice similarity coefficient of 0.665 for segmentation, and a classification performance with an area under the curve at receiver operating characteristics analysis of 0.978 , a sensitivity of 0.946 , and a specificity of 0.936. Segmentation but not classification performance of DCE-MRI improved with information from DWI and FDGPET. Feature ranking revealed that kinetic and spatio-temporal texture features had the highest contribution for lesion classification. ${ }^{18} \mathrm{~F}-\mathrm{FDG}-\mathrm{PET}$ and morphologic features were less predictive.
\end{abstract}

Conclusion: Our CAD enables the assessment of the relevance of mPPET/MRI features on segmentation and classification accuracy. It may aid as a novel computational tool for exploring different modalities/features and their contributions for the detection and classification of breast lesions.

Keywords: Diagnosis (computer-assisted), Breast neoplasms, Magnetic resonance imaging, Machine learning, Positron-emission tomography

\footnotetext{
*Correspondence: georg.langs@meduniwien.ac.at

${ }^{+}$Wolf-Dieter Vogl and Katja Pinker contributed equally to this work.

${ }^{1}$ Computational Imaging Research Laboratory, Department of Biomedical

Imaging and Image-guided Therapy, Medical University Vienna, Waehringer

Guertel 18-20, 1090 Vienna, Austria

Full list of author information is available at the end of the article
} 


\section{Key points}

- The positron emission tomography/magnetic resonance imaging (PET/MRI) computer-aided segmentation and diagnosis (CAD) system automatically detects, segments, and classifies breast lesions.

- Automatic lesion segmentation was accurate and improved with information from all modalities.

- A small number of features mainly from dynamic contrast-enhanced MRI achieves high classification accuracies.

- The PET/MRI-CAD system allows exploring the value of different imaging modalities and features.

\section{Background}

Breast cancer is the most common cancer and the second most common cause of mortality from cancer in women [1]. Early detection and precise diagnosis are important for effective treatment [2], and breast imaging plays a pivotal role in the detection, characterisation, and staging of breast cancer. Recently, multimodal, multiparametric imaging $(\mathrm{mpI})$ including dynamic contrast-enhanced magnetic resonance imaging (DCE-MRI), diffusion-weighted imaging (DWI), and positron emission tomography (PET) has been investigated for an improved differentiation of benign and malignant breast lesions [3]. Such imaging constitutes complex protocols but is promising for a more comprehensive measurement of morphology (MRI), neoangiogenesis (DCE-MRI), tumour metabolism (PET), and microstructure (DWI) in cancerous and benign tissue [3] (Fig. 1).

Due to the increased complexity of the information captured by mpI, computational approaches that enable the quantitative assessment of multivariate measurements have been gaining relevance. Recently, computer-aided detection and diagnosis systems have been proposed to reduce inter- and intra-reader variability and to aid radiologists in the detection and diagnosis of breast cancer [4]. These systems are able to analyse large amounts of imaging data in a short time, detect and visualise complex correlations and patterns, and provide objective and repeatable measurements [5] to increase the accuracy of diagnosis [6]. Computer-aided detection (CADe) systems assist radiologists in localising suspicious regions in medical images, whereas computer-aided diagnosis (CADx) systems support the radiologist in the diagnosis of suspicious regions by providing and analysing information extracted from these regions [7]. These systems show potential to be advantageous in the current clinical scenario [7] where despite guidelines for DCE-MRI, such as the Breast Imaging-Reporting and Data System (BI-R$\mathrm{ADS}^{\circ}$ ) MRI lexicon [8], inter- and intra-reader variability remains an issue and the human analysis of complex relationships observed in images and the underlying disease remains limited [9].
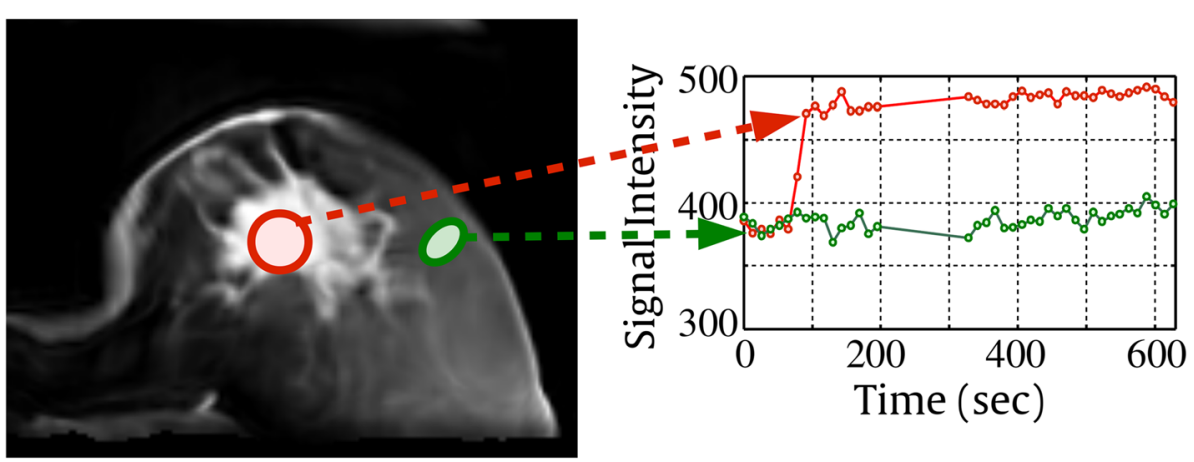

DCE-MRI
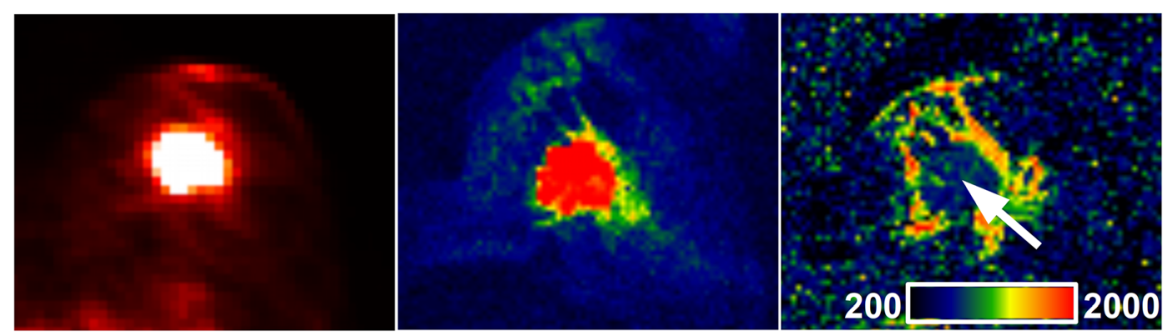

$\left[{ }^{18} \mathrm{~F}\right]-$ FDG PET DWI $\left(\mathrm{b}=850 \mathrm{sec} / \mathrm{mm}^{2}\right) \quad$ DWI ADC

Fig. 1 Image modalities covering the lesion. Top: DCE-MRI time-signal intensity curve extracted from an ROI within the lesion (red) and from normal tissue (green), illustrating the contrast enhancement within the lesion. Bottom from left to right: ${ }^{18}$ F-FDG-PET, DWI, and ADC map. Note the decreased ADC values in the lesion area (white arrow) 
As yet, the information provided by individual imaging techniques as part of mpI remains poorly understood. To identify the diagnostically relevant parameters captured across DCE-MRI, DWI, and ${ }^{18} \mathrm{~F}$-fluorodeoxyglucose $\left({ }^{18} \mathrm{~F}\right.$-FDG)-PET, we propose a novel automated data-driven approach: a combined breast lesion segmentation and classification system for $\mathrm{mpI}$ data where the system automatically identifies the information in the imaging data that contribute to an accurate segmentation and classification.

\section{Methods}

\section{Patients}

The data used in this retrospective analysis was acquired from an institutional review board-approved prospective, single-institution study [25]. All patients gave written informed consent. At the time of the prospective study, only prototypic PET/MRI scanners were in existence and these were not available at the study centre. Thus, 46 patients were included in this prospective study in which MRI and a combined computed tomography (CT) $/{ }^{18}$ F-FDG-PET were acquired. All tumours were histopathologically verified. In our retrospective analysis, the CT image was used only as morphologic information for the registration and was purposely not part of segmentation and classification. After applying our automatic CT to MRI registration method, as described below, twelve patients had to be removed from analysis due to registration errors. All excluded cases were patients with large breasts that were considerably compressed, or deformed, in one of the modalities during image acquisition. Misalignments were detected visually by overlaying MRI and CT images. From the remaining 34 patients, 12 had benign lesions and 22 had malignant lesions (2 patients had multifocal or multicentric cancer). Characteristics of the lesions are listed in Table 1.

\section{Image acquisition}

Patients underwent 3T MRI (Tim Trio, Siemens, Erlangen, Germany) in prone position using a four-channel

Table 1 Patient and breast lesion characteristics

\begin{tabular}{ll}
\hline Patients $(n=34)$ & $51.36 \pm 12.26$ (24-78) \\
Age, years, mean \pm SD (range) & \\
Lesions $(n=36)$ & $1.8 \pm 1.1(0.6-4.9)$ \\
Diameter, cm, mean \pm SD (range) & \\
Lesion type & $24(66.7 \%)$ \\
$\quad$ Malignant & $19(52.8 \%)$ \\
$\quad$ Invasive ductal carcinoma & $3(8.3 \%)$ \\
$\quad$ Invasive lobular carcinoma & $2(5.6 \%)$ \\
$\quad$ Ductal carcinoma in situ & $12(33.3 \%)$ \\
Benign & $36(100.0 \%)$ \\
Total
\end{tabular}

$S D$ standard deviation breast coil (InVivo, Orlando, FL, USA) and a combined whole-body PET/CT in-line system (Biograph 64 TruePoint ${ }^{\circ}$; Siemens, Erlangen, Germany) in prone position.

For DCE-MRI a split dynamics protocol that combined high-spatial and high-temporal resolution was used [11]. First, a high spatial resolution, pre-contrast coronal T1-weighted turbo three-dimensional fast low angle shot (FLASH) sequence with water-excitation and fat-suppression was acquired with matrix $320 \times 320 \times 120$ and 1 -mm isotropic voxel (DCE-MRI pre-contrast imaging, $\mathbf{I}_{\text {dce-pre }}$ ). Subsequently, a DCE coronal T1-weighted volumetric interpolated breath-hold-examination (VIBE) sequence with 17 acquisitions (13.2 s per acquisition) was acquired with matrix $192 \times 192 \times 72 \mathrm{~mm}$ and $1.7-\mathrm{mm}$ isotropic voxel (DCE-MRI, $\mathbf{I}_{\text {dce }}$ ). Seventy-five seconds after the beginning of the sequence, gadoterate meglumine (Gd-DOTA, Dotarem ${ }^{\circ}$, Guerbet, Paris, France) was injected as a bolus at a dose of $0.1 \mathrm{mmol} / \mathrm{kg}$ at a rate of $4 \mathrm{~mL} / \mathrm{s}$ and followed by a $20-\mathrm{mL}$ saline flush at the same injection rate. Then, a FLASH sequence was acquired to capture the peak enhancement of lesions (DCE-MRI peak-contrast imaging, $\left.\mathbf{I}_{\text {dce-peak }}\right)$, followed by a VIBE sequence with the same parameters above described. Finally, a FLASH sequence with the same parameters above described was acquired (DCE-MRI post-contrast imaging, $\mathbf{I}_{\text {dce-post }}$ ) to depict delayed enhancement lesion morphology. DWI sequences were acquired in the same session, with $b$ values of 50 and $850 \mathrm{~s} / \mathrm{mm}^{2}$, resulting into two datasets, $\mathbf{I}_{\mathrm{dwi}}$ bo and $\mathbf{I}_{\mathrm{dwi}}$ b850, as well as the derived apparent diffusion coefficient (ADC) mapping, $\mathbf{I}_{\text {adc }}$ [12] (matrix $172 \times 86 \times 24$, pixel $2.09 \times 2.09 \mathrm{~mm}$, slice thickness $5.5 \mathrm{~mm}) .{ }^{18} \mathrm{~F}$-FDG-PET (matrix $168 \times 168 \times 74$, pixel $4 \times 4 \mathrm{~mm}$, slice thickness 3 $\mathrm{mm}$ ) and CT images (matrix $512 \times 512 \times 74$, pixel $1.37 \times$ $1.37 \mathrm{~mm}$, slice thickness $3 \mathrm{~mm}$ ) of the thorax were acquired in a hybrid PET/CT scanner and were aligned by the scanner software.

\section{CAD pipeline}

We developed a novel automated data-driven combined CADx system for mpI data with MRI and PET. The system enabled automatic detection and segmentation of potentially cancerous regions and classified lesions as benign or malignant. The algorithm first aligned multimodal breast imaging data from DCE-MRI, DWI, and ${ }^{18}$ F-FDG PET non-rigidly, and segmented the breast. Then, the system extracted local textural, kinetic, and intensity-based image features from the fused information and detected and classified lesions using a random forest (RF) classifier [10]. Figure 2 shows the overview of the proposed CAD pipeline.

\section{Alignment}

To collect information at individual positions across modalities, all images were aligned to $\mathbf{I}_{\text {dce-pre }}$ serving as 


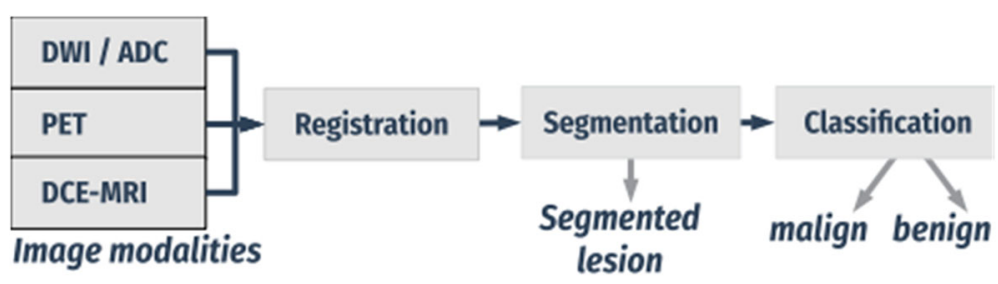

Fig. 2 Overview of the CAD pipeline based on multimodal and mpl features

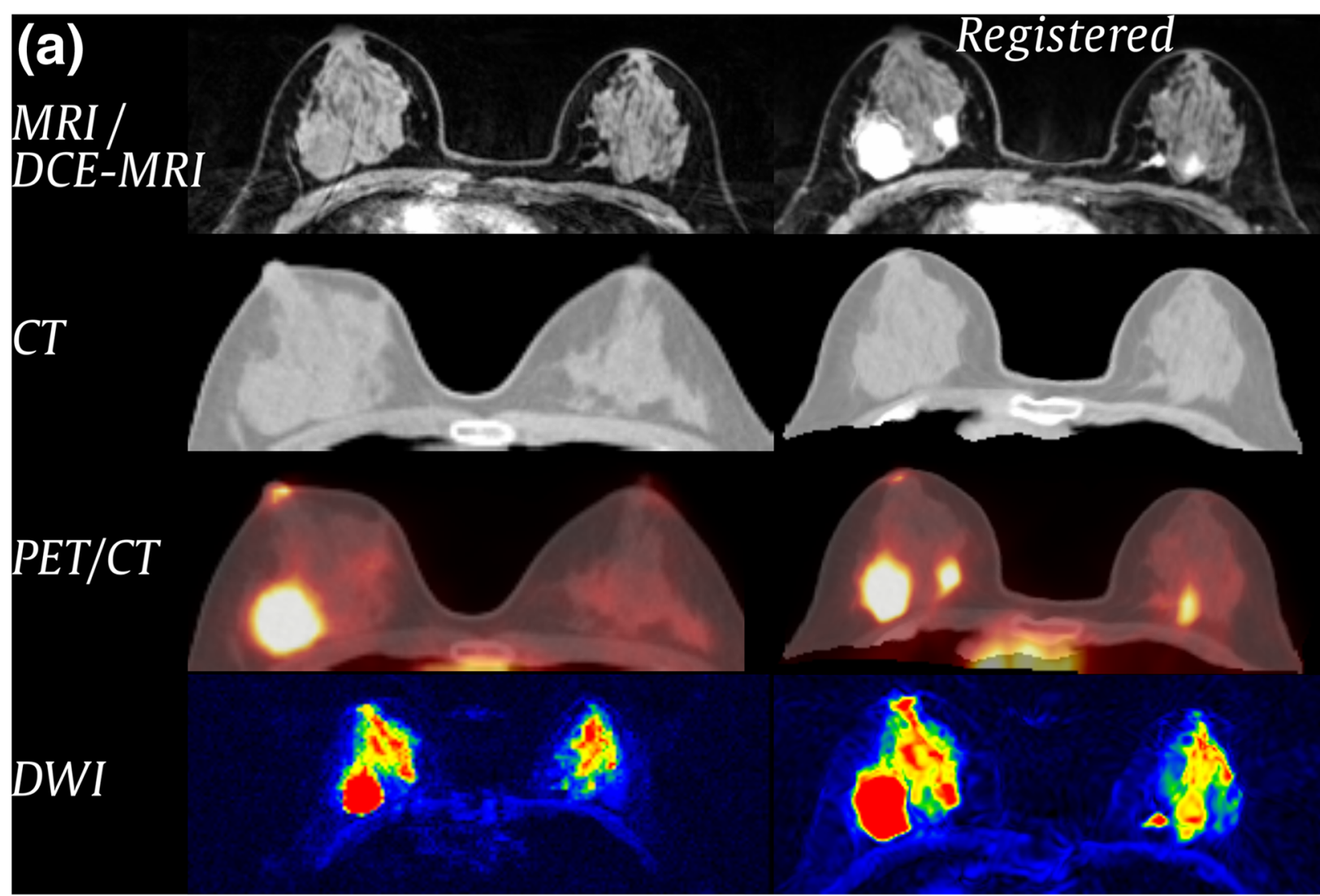

(b)

Probability Map

Segmentation

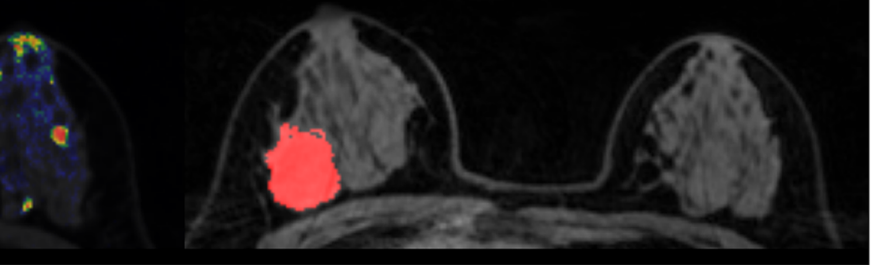

Fig. 3 Results of the (a) registration and (b) segmentation process for one patient. a First row: Reference $I_{d c e-p r e ~}$ and registered $I_{d c e-p o s t}$. Second row: Ict unregistered/registered. Third row: $I_{\text {pet }}$ image unregistered/registered, fused with the corresponding CT image. Fourth row: $I_{d w i}$ bo unregistered/registered. b Probability map obtained from voxel-wise classification overlaid on the MR pre-contrast image (left) and final segmentation after applying a threshold and post-processing (right) 
Table 2 Features extracted for each voxel $(\mathbf{x})$ within the breast (M)

\begin{tabular}{|c|c|c|c|}
\hline $\begin{array}{l}\text { Feature } \\
\text { group }\end{array}$ & Description & Definition & $\begin{array}{l}\text { Number of features } \\
\text { per voxel }\end{array}$ \\
\hline $\mathbf{f}_{\mathrm{dce}}$ & $\begin{array}{l}\text { DCE-MRI intensity values for each frame } \\
\text { of the DCE-MRI time series }\end{array}$ & $\begin{array}{l}\mathbf{f}_{\mathrm{dce}}(\mathbf{x}):=\left\{\mathbf{I}_{\mathrm{dce}}(\mathbf{x}, i), j \leq i \leq j+25\right\} \text {, where } j \text { is the first frame with contrast- } \\
\text { enhancement and } i \text { is the frame number in the DCE sequence }\end{array}$ & 26 \\
\hline $\mathbf{f}_{\delta \text { dce }}$ & $\begin{array}{l}\text { Difference of DCE-MRI intensity values } \\
\text { between two frames with distance } 2\end{array}$ & $\begin{array}{l}\mathbf{f}_{\delta \text { dce }}(\mathbf{x}):=\left\{\frac{\boldsymbol{I}_{\text {dce }}(\mathbf{x}, i+2)-\boldsymbol{I}_{\text {dce }}(\mathbf{x}, i)}{t_{i+2}-t_{i}} \mid j \leq i \leq j+25\right\} \text {, where } t_{i} \text { is the time point of } \\
\text { acquisition of frame } i\end{array}$ & 25 \\
\hline $\mathbf{f}_{\text {nsumdce }}$ & Normalised sum of DCE-MRI intensities & $\mathbf{f}_{\text {nsumdce }}(\mathbf{x}):=\frac{\mathbf{f}_{\text {sumdce }}(\mathbf{x})}{\text { maxy }_{y \in M}\left(\mathbf{f}_{\text {sumdce }}(y)\right)}$ where $\mathbf{f}_{\text {sumdce }}(\mathbf{x}):=\sum_{i=j}^{j+25} \mathbf{I}_{\text {dce }}(\mathbf{x}, i)$ & 1 \\
\hline $\mathbf{f}_{\mathrm{mri}}$ & $\begin{array}{l}\text { Intensity values for high-resolution MRI: } \\
\mathbf{I}_{\text {dcepre, }} \mathbf{I}_{\text {dcepeak, }} \text { and } \mathbf{I}_{\text {dcepost }}\end{array}$ & $\mathbf{f}_{\text {mri }}(\mathbf{x}):=\left\{\mathbf{I}_{\text {dcepre }}(\mathbf{x}), \mathbf{I}_{\text {dcepeak }}(\mathbf{x}), \mathbf{I}_{\text {dcepost }}(\mathbf{x})\right\}$ & 3 \\
\hline $\mathbf{f}_{\delta \mathrm{mri}}$ & $\begin{array}{l}\text { Difference in intensity values for high- } \\
\text { resolution MR images }\end{array}$ & $\begin{array}{l}\mathbf{f}_{\delta \text { mri }}(\mathbf{x}):=\left\{\mathbf{I}_{\text {dcepost }}(\mathbf{x})-\mathbf{I}_{\text {dcepre }}(\mathbf{x}), \mathbf{I}_{\text {dcepeak }}(\mathbf{x})-\mathbf{I}_{\text {dcepre }}(\mathbf{x}), \mathbf{I}_{\text {dcepost }}(\mathbf{x})-\right. \\
\left.\mathbf{I}_{\text {dcepeak }}(\mathbf{x})\right\}\end{array}$ & 3 \\
\hline $\mathbf{f}_{\mathrm{dwi}}$ & DWI intensity value & $\mathbf{f}_{\mathrm{dwi}}(\mathbf{x}):=\left\{\mathbf{I}_{\mathrm{dwi}} b 0(\mathbf{x}), \mathbf{I}_{\mathrm{dwi} b 850}(\mathbf{x}), \mathbf{I}_{\mathrm{adc}}(\mathbf{x})\right\}$ & 3 \\
\hline $\mathbf{f}_{\text {pet }}$ & PET intensity value & $\mathbf{f}_{\text {pet }}(\mathbf{x}):=\left\{\boldsymbol{I}_{\text {pet }}(\mathbf{x})\right\}$ & 1 \\
\hline
\end{tabular}

$D C E-M R I$ dynamic contrast-enhanced magnetic resonance imaging, $D W I$ diffusion-weighted imaging, $P E T$ positron emission tomography

reference coordinate system. Images were registered with the software package Advanced Normalisation Tools (ANTs) [13] using an affine transformation with mutual information as the similarity metric, followed by a non-rigid deformation with symmetric normalisation (SyN) [13] and windowed normalised cross-correlation as a similarity metric (Fig. 3a). As $\mathbf{I}_{\text {pet }}$ does not provide morphologic information, we registered the corresponding CT image to $\mathbf{I}_{\text {dce-pre }}[14]$ and subsequently applied the obtained transformation on $\mathbf{I}_{\text {pet }}$.

\section{Lesion segmentation}

We treated lesion segmentation as a voxel-wise classification problem, where a machine learning algorithm assigned a binary label 1 (lesion) or 0 (non-lesion) to each voxel based on imaging features extracted at that location. As ground truth for training and validation, we used manual expert radiologist (with 3 years of experience) annotations performed on the registered $\mathbf{I}_{\text {dce-peak }}$ or $\mathbf{I}_{\text {dce-post }}$, depending on where the lesion borders were better visible. Annotations were validated by a second expert radiologist with 9 years of experience.

All computations were restricted to the breast area, which was segmented using an intensity-based growing region algorithm [15]. All MRI intensity values were standardised to zero mean and unit standard-deviation estimated from the breast area on the pre-contrast images, $\mathbf{I}_{\text {dce-pre }}$ and $\mathbf{I}_{\text {dce }}$. We computed intensity features from all imaging data, from changes of the contrast over time and the summed up contrast in the DCE-MRI sequence as specified in Table 2 .

An RF classifier model was trained on features extracted from 1000 randomly selected samples per class and patient. The trained model was then used to predict the segmentation label for a new patient who was not part of the training data set for each voxel $x$ of the breast based on the computed features (Fig. 3b).

\section{Lesion classification}

After segmentation, the lesion was classified as either benign or malignant based on features extracted per lesion. Intensity-based, kinetic, morphological, and textural features were considered to train a lesion class prediction model, and the obtained model was used to predict malignancy for lesions in the new patient who was not part of the training data set.

Intensity-based features were calculated from DCE-MRI, DWI ADC, and the ${ }^{18}$ F-FDG-PET map. We tackled the lesion inhomogeneities in the contrast enhancement of DCE-MRI by the method described by Chen et al. [16], where the signal-to-time curves within a lesion were clustered by the fuzzy c-means algorithm and the curve with highest contrast enhancement rate, the characteristic kinetic curve, was chosen for classification. We used the 25 time points beginning with contrast enhancement $\left(f_{\text {lckc }}\right)$ and the change over time $\left(f_{\text {lockc }}\right)$ calculated by forward difference (four frames) as intensity features. Analogously, $\mathbf{I}_{\text {adc }}$ and $\mathbf{I}_{\text {pet }}$ intensities were partitioned into five clusters and the cluster centre with the lowest $\mathrm{ADC}$ value and the highest ${ }^{18}$ F-FDG uptake were used as features $\mathbf{f}_{\text {l-adc }}$ and $\mathbf{f}_{\text {l-pet}}$.

To capture contrast enhancement kinetics, we fitted an asymmetric generalised logistic function as regression function multiplied with an exponential term to the characteristic kinetic curve:

$$
\begin{aligned}
C\left(t, G, \alpha, \tau, t_{1 / 2}, \beta, k\right)=G & \cdot\left(1-\frac{1}{\left(1+\left(2^{\alpha}-1\right) \cdot \exp \left(\frac{1}{\tau} \cdot\left(t-t_{1 / 2}\right)\right)\right)^{1 / \alpha}}\right) \\
\cdot & \exp \left(\beta \cdot t^{k}\right)
\end{aligned}
$$

where $G$ defines the scaling, $\alpha$ the asymmetry parameter, 
$\tau$ the steepness, and $t_{1 / 2}$ the time of half maximum of the sigmoid function; $k$ defines the terminal slope and $\beta$ scaling factor of the exponential term (Additional file 1: Figure S1). We used the parameters $\alpha, \tau, \beta$, and $k$ as features $\left(f_{\text {lkinetic }}\right)$. In addition, we computed summary measures of the curve within a 7-min interval, beginning at start of contrast enhancement: area under the curve $(A u C)$, maximum enhancement $\left(C_{\max }\right)$, time to maximum enhancement $\left(T_{\max }\right)$, time to half maximum enhancement $\left(T_{1 / 2}\right)$, and maximum analytical derivative $\frac{\delta C}{\delta t}$ of the regression function $C(t)(M D E R)$.

To obtain textural features, $\mathbf{f}_{\text {-texture, }}$ we used a volumetric texture analysis approach based on grey-level co-occurrence matrix (GLCM) and Haralick texture features $[17,18]$. We computed the GLCM with 128 Gy-value bins and 26 neighbours within the lesion and used its 13 s-order statistics [17]. $\mathbf{f}_{\text {l-tex-pre }}, \mathbf{f}_{\text {l-tex-peak }}$, and $\mathbf{f}_{\text {l-tex-post }}$ contained the Haralick features obtained from the $\mathbf{I}_{\text {dce-pre, }}$ $\mathbf{I}_{\text {dce-peak }}$, and $\mathbf{I}_{\text {dce-post }}$ intensity values, respectively.

In addition to the spatial texture analysis, we used a novel temporal texture analysis inspired by the works of Agner et al. [19] and Woods et al. [20]. With this analysis, we characterised the temporal properties of contrast uptake within a lesion, e.g., homogeneity of contrast uptake. To compute the GLCMs, we considered voxel pairs at the same spatial position $\mathbf{x}$ but at different time points in the contrast enhancement. We computed the Haralick features from pixel pairs from $\left(\mathbf{I}_{\text {dce-pre, }}, \mathbf{I}_{\text {dce-peak }}\right)$, ( $\mathbf{I}_{\text {dce-pre, }}$, $\left.\mathbf{I}_{\text {dce-post }}\right)$, and ( $\left.\mathbf{I}_{\text {dce-peak }}, \mathbf{I}_{\text {dce-post }}\right)$, resulting in the feature vectors $\mathbf{f}_{\text {l-tex-peak/pre, }}, \mathbf{f}_{1 \text {-tex-post/pre, }}$ and $\mathbf{f}_{\text {l-tex-post/peak }}$.

To obtain morphological feature candidates, $f_{\text {lmorph }}$, we used shape descriptors, as utilised previously in the literature $[19,21,22]$. Definitions of the shape descriptors are given in Additional file 1: Table S1.

\section{Evaluation of lesion segmentation and classification}

To evaluate lesion segmentation, we performed experiments in a leave-one-out cross-validation (LOOCV) fashion, training the segmentation algorithm and feature rankings on all but one example, and applying it to the remaining example not included in the training. The quality of the segmentation was measured on a pixel level by comparing the predicted segmentation with the manually annotated data using Dice similarity coefficient (DSC) [23] as a similarity measure and sensitivity (truepositive rate) describing the probability of detection. As RF provide probabilities, we determined the RF threshold as the one that maximises DSC on the training set. Overall performance was obtained by computing the mean of all test DSC scores.

To evaluate lesion classification, we classified lesions into the two classes: benign and malignant. Evaluation was performed in an LOOCV fashion for both ranking the features and determining accuracy. Accuracy was reported as receiver operating characteristic (ROC) area under the curve (AUC) and sensitivity/specificity. The RF threshold was chosen within the training set as the one maximising the $F_{1}$ score, which is the harmonic mean of precision and sensitivity. All experiments were repeated 20 times, and averages for AUC and sensitivity/ specificity are reported. To study the impact of segmentation accuracy on classification, we performed classification on both manually delineated lesions and automatically segmented lesions.

In a post-processing step, false-positive blobs were removed by computing connected-components from the segmentations using a six-neighbourhood, and only blobs that partially overlapped with the manual annotation were selected. This step mimics the manual selection of a suspicious region that a radiologist wants to investigate further. For the two benign cases where the lesion was not detected, manual segmentation was used instead of the automatic segmentation. This post-processing step allowed us to evaluate classification accuracy independent of the segmentation performance.

\section{Evaluation of feature contribution}

We then evaluated the contribution of features collected across the mpI data and ranked their contribution to segmentation and classification based on two measures: (1) RF Gini importance (GI) [10] and (2) minimumredundancy-maximum-relevance (mRMR) [24]. The GI measures the average amount of information gain using the Gini index splitting criterion during RF training and ranks the contribution of each feature as part of a multivariate pattern. If features are redundant but informative, it ranks all of them highly [25]; the mRMR provides a ranking based on relevance and redundancy of the features. Then, we successively increased the number of features for training and validation, beginning with the top-ranked feature, and measured the performance of each model, thus allowing us to assess the contribution of each individual feature in a multimodal, multiparametric setup. In addition, the benefits of multiparametric and multimodal features were evaluated by training models using only DCE-MRI features and combined DCE-MRI, DWI, and/or ${ }^{18}$ F-FDG PET features.

\section{Results}

\section{Lesion segmentation}

We report in Table 3 and illustrate in Additional file 1: Figure S2 the performance of the models showing the highest DSC for Gini importance and mRMR feature selection with and without multiparametric features. The model with mRMR feature selection and the top eight features showed a mean/median DSC of 0.665 / 
Table 3 Automatic segmentation performance in terms of DSC and sensitivity

\begin{tabular}{llll}
\hline Features & DSC mean \pm SD/median \pm IQR & Sensitivity mean \pm SD/median \pm IQR & Number of features \\
\hline Gl & $0.607 \pm 0.238 / 0.691 \pm 0.255$ & $0.661 \pm 0.234 / 0.721 \pm 0.263$ & 2 \\
Gl without PET & $0.608 \pm 0.281 / 0.722 \pm 0.419$ & $0.739 \pm 0.290 / 0.876 \pm 0.238$ & 11 \\
Gl without DWI & $0.573 \pm 0.290 / 0.708 \pm 0.445$ & $0.669 \pm 0.324 / 0.825 \pm 0.445$ & 8 \\
Gl without DWI, without PET & $0.577 \pm 0.277 / 0.665 \pm 0.419$ & $0.658 \pm 0.343 / 0.813 \pm 0.597$ & 25 \\
mRMR & $\mathbf{0 . 6 6 5} \pm 0.236 / \mathbf{0 . 7 5 7} \pm 0.189$ & $0.743 \pm 0.267 / 0.836 \pm 0.269$ & 8 \\
mRMR without PET & $0.618 \pm 0.272 / 0.749 \pm 0.413$ & $\mathbf{0 . 7 4 8} \pm 0.277 / 0.873 \pm 0.307$ & 7 \\
mRMR without DWI & $0.601 \pm 0.268 / 0.701 \pm 0.377$ & $0.686 \pm 0.328 / 0.801 \pm 0.538$ & 7 \\
mRMR without DWI, without PET & $0.584 \pm 0.300 / 0.710 \pm 0.393$ & $0.613 \pm 0.338 / 0.784 \pm 0.496$ & 8
\end{tabular}

DSC Dice similarity coefficient, DWI diffusion-weighted imaging, IQR interquartile range, GI Gini Importance, mRMR minimum-redundancy-maximum-relevance, $P E T$ positron emission tomography, SD standard deviation. Values presented in bold are the highest values

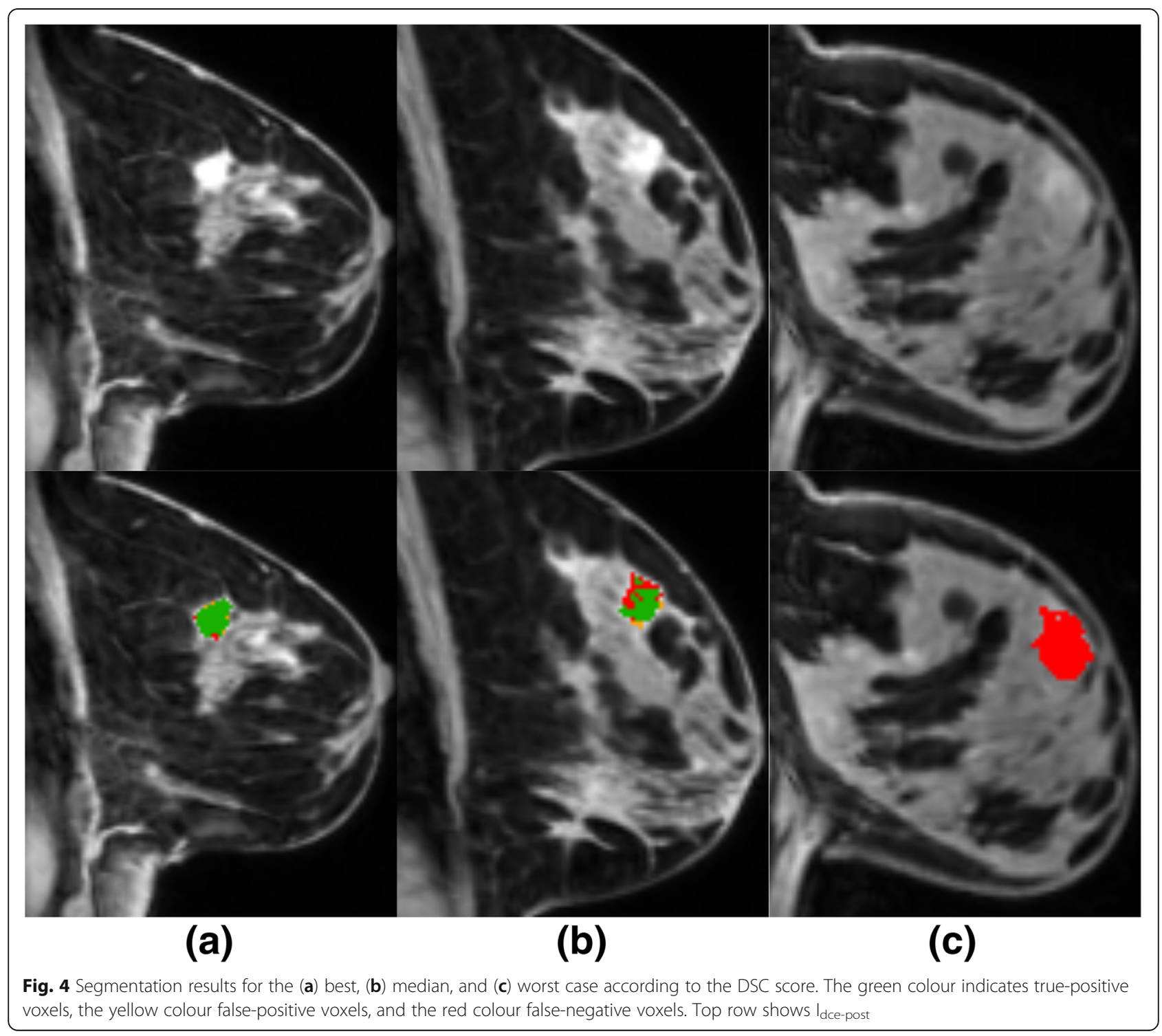



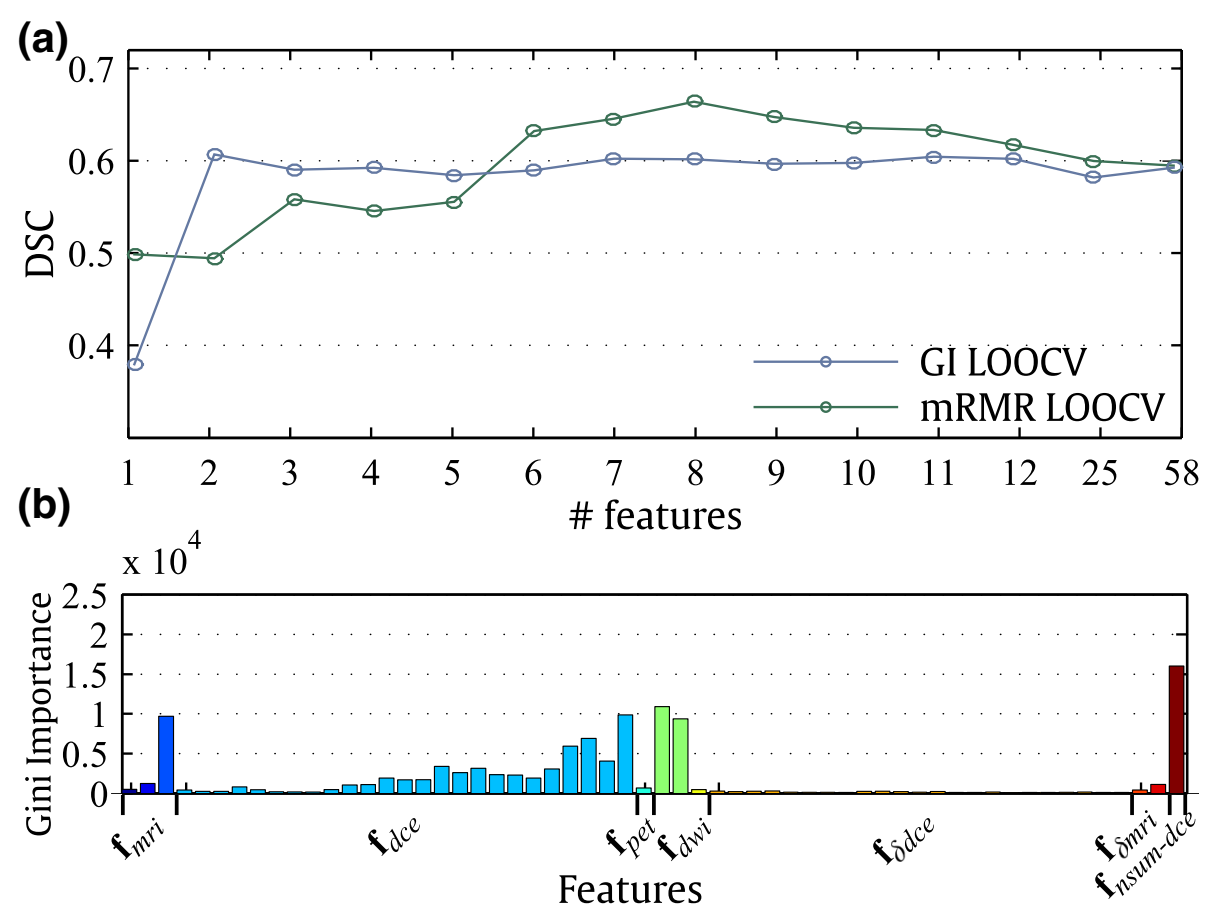

Fig. 5 Feature ranking and its influence on segmentation performance. a The mean DSC, using a successively increasing number of top-ranked features according to RF GI and mRMR ranking. $\mathbf{b}$ Gl feature ranking of the segmentation features. The four top-ranked features are labelled in the figure

0.757. Here, DSC benefited from multiparametric features, showing a reduced mean DSC of 0.601 without DWI, 0.618 without PET, and 0.584 with only DCE-MRI features. The model with GI feature selection showed a lower performance with a DSC of 0.607 compared with the model with mRMR feature selection. Here, DSC also benefitted from multiparametric features, showing a reduced DSC of 0.577 with only DCE-MRI features. The improvement in segmentation accuracy for multiparametric features mainly resulted

Table 4 Top-ranked segmentation features according to Gini importance and minimum-redundancy-maximum-relevance

\begin{tabular}{|c|c|c|}
\hline Rank & Gini importance & Minimum-redundancy-maximum-relevance \\
\hline 1 & $\mathbf{f}_{\text {nsum-dce }}$ & $I_{\text {dce-post }}$ \\
\hline 2 & $\mathbf{f}_{\mathrm{dwi}} \# 1$ & $\mathbf{f}_{\delta \mathrm{dce}} \# 16$ \\
\hline 3 & $\mathbf{f}_{\text {dce }} \# 25$ & $\mathbf{f}_{\mathrm{dwi}} \# 2$ \\
\hline 4 & $I_{\text {dce-post }}$ & $\mathbf{f}_{\mathrm{pet}}$ \\
\hline 5 & $\mathbf{f}_{\mathrm{dwi}} \# 2$ & $\mathbf{f}_{\text {nsum-dce }}$ \\
\hline 6 & $\mathbf{f}_{\text {dce }} \# 23$ & $\mathbf{f}_{\mathrm{dwi}} \# 1$ \\
\hline 7 & $\mathbf{f}_{\text {dce } \# 22}$ & $\mathbf{f}_{\delta m r i} \# 1$ \\
\hline 8 & $\mathbf{f}_{\mathrm{dce}} \# 24$ & $\mathbf{f}_{\text {ठdce }} \# 23$ \\
\hline 9 & $\mathbf{f}_{\text {dce }} \# 15$ & $\mathbf{f}_{\delta \mathrm{mri}} \# 2$ \\
\hline 10 & $\mathbf{f}_{\mathrm{dce}} \# 17$ & $\mathbf{f}_{\delta \text { dce }} \# 20$ \\
\hline
\end{tabular}

Numbers next to each feature (\#) indicate the frame index in the dynamic contrast-enhanced magnetic resonance imaging. DSC Dice similarity coefficient. See text for the abbreviations subscripted after $\mathbf{f}$ or $\mathbf{I}$ from reducing false-positive cases, such as vessels and enhancing parenchymal areas. Overall, for this dataset, we had a detection rate of $22 / 22(100 \%)$ for malignant lesions and of $10 / 12(83.3 \%)$ for benign lesions. As shown in Fig. 4, the missed benign lesions had a very low contrast uptake and thus were missed by the prediction models.

The performance of the GI and mRMR feature selection models with an increasing number of highest-ranked features is shown in Fig. 5a. The performance of the GI feature selection model peaked at only three features whereas the performance of the mRMR feature selection model peaked at six features. Table 4 shows the ranking of the features according to GI and mRMR. Both algorithms ranked $\mathbf{f}_{\text {dwi }}, \mathbf{f}_{\text {nsum-dce, }}$ and $\mathbf{I}_{\text {dce-post }}$ highly. However, mRMR tended to pick more varied features than GI, where GI selected six potentially correlated features from $\mathbf{f}_{\text {dce }}$ as part of the top 10 features. The features capturing changes in the contrast, $\mathbf{f}_{\delta \mathrm{dce}}$ and $\mathbf{f}_{\delta \mathrm{mr}}$, received a lower ranking in GI (see also Fig. 5b) compared with mRMR.

\section{Lesion classification}

In Table 5, we list the results for the models showing the highest ROC AUC score after GI and mRMR feature selection. Overall, for manually annotated lesions, mRMR feature selection yielded the highest AUC (0.978) using only two features, with a sensitivity of $94.6 \%$ and specificity of $93.6 \%$ for identifying 
Table 5 Classification results for differentiation of malignant and benign lesions for manually annotated lesions and automatic segmented lesions using automatic feature selection

\begin{tabular}{|c|c|c|c|c|c|c|}
\hline \multirow[t]{2}{*}{ Feature selection method } & \multicolumn{3}{|l|}{ Manual annotation } & \multicolumn{3}{|c|}{ Automatic segmentation } \\
\hline & $\mathrm{AUC}($ mean $\pm \mathrm{SD})$ & $\begin{array}{l}\text { Sensitivity/ } \\
\text { specificity }\end{array}$ & $\begin{array}{l}\text { Number of } \\
\text { features }\end{array}$ & $\mathrm{AUC}($ mean $\pm \mathrm{SD})$ & $\begin{array}{l}\text { Sensitivity/ } \\
\text { specificity }\end{array}$ & $\begin{array}{l}\text { Number of } \\
\text { features }\end{array}$ \\
\hline GI LOOCV & $0.949 \pm 0.019$ & $0.920 / 0.868$ & 4 & $0.771 \pm 0.040$ & $0.961 / 0.482$ & 100 \\
\hline GI LOOCV without PET & $0.946 \pm 0.002$ & $0.924 / 0.859$ & 4 & $0.771 \pm 0.040$ & $0.972 / 0.486$ & 75 \\
\hline Gl LOOCV without DWI & $0.949 \pm 0.015$ & $0.915 / 0.873$ & 4 & $0.754 \pm 0.035$ & $0.983 / 0.427$ & 75 \\
\hline GI LOOCV without DWI, without PET & $0.944 \pm 0.018$ & $0.922 / 0.868$ & 4 & $0.755 \pm 0.033$ & $0.976 / 0.409$ & 75 \\
\hline mRMR LOOCV & $0.978 \pm 0.008$ & $0.946 / 0.936$ & 2 & $0.858 \pm 0.013$ & $0.941 / 0.773$ & 3 \\
\hline mRMR LOOCV W/O PET & $0.975 \pm 0.010$ & $0.957 / 0.918$ & 2 & $0.856 \pm 0.018$ & $0.948 / 0.736$ & 3 \\
\hline mRMR LOOCV w/o DWI & $0.977 \pm 0.006$ & $0.954 / \mathbf{0 . 9 5 0}$ & 2 & $0.857 \pm 0.017$ & $0.943 / 0.745$ & 3 \\
\hline mRMR LOOCV w/o DWI, PET & $0.973 \pm 0.010$ & $0.950 / 0.927$ & 2 & $\mathbf{0 . 8 6 1} \pm 0.009$ & $0.941 / 0.755$ & 3 \\
\hline
\end{tabular}

$A U C$ area under the curve at receiver operating characteristic analysis, DCE-MRI dynamic contrast-enhanced magnetic resonance imaging, DWI diffusion-weighted imaging, GI Gini importance, LOOCV leave-one-out cross-validation, mRMR minimum-redundancy-maximum-relevance, $P E T$ positron emission tomography, SD standard deviation. Values presented in bold are the highest values

malignant lesions. When automatic segmentation was used, the highest ROC AUC was 0.861 including only three DCE-MRI features. mRMR feature selection showed a better AUC performance than GI, both for manual annotation (0.978 versus 0.949$)$ and automatic segmentation (0.861 versus 0.771$)$.

The performance of the GI and mRMR feature selection models with an increasing number of highest-ranked features is shown in Fig. 6a. The mRMR feature selection model peaked at only two features whereas the GI feature selection model peaked at four features, with a subsequent decrease in AuC performance. A closer look at the ranking of the features (Table 6 and Fig. 6b) indicates that features from the pool of kinetic $\left(\mathbf{f}_{\text {l-kinetic }}\right)$ and textural $\left(\mathbf{f}_{1 \text {-texture }}\right)$ features were top-ranked by GI and mRMR models. Morphologic $\left(\mathbf{f}_{\text {l-morph }}\right)$ and PET $\left(\mathbf{f}_{\text {l-pet }}\right)$ features received a low ranking by GI and mRMR models. The DWI ADC feature $\left(\mathbf{f}_{1 \text {-adc }}\right)$ was ranked as an important feature by GI in automatic segmentation only.
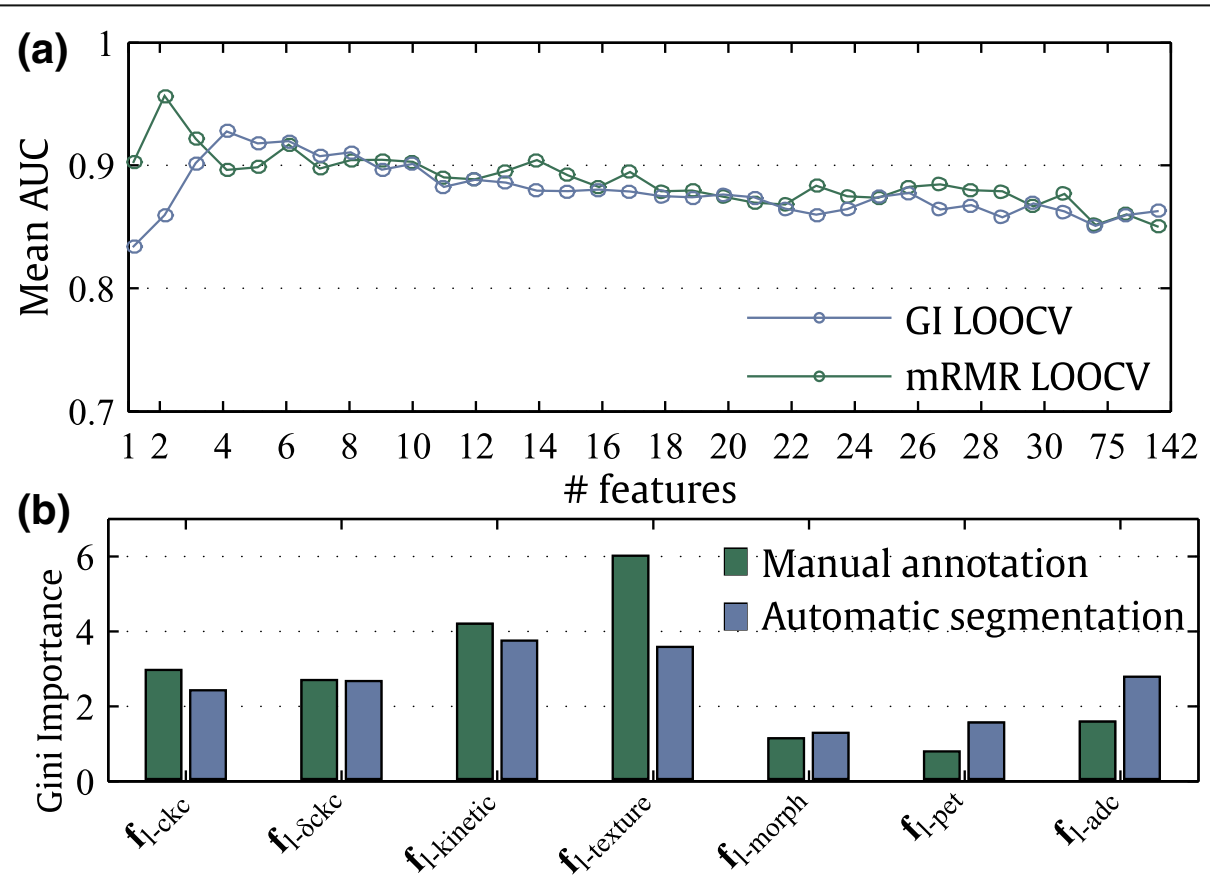

Fig. 6 Feature ranking and its influence on classification performance. a Mean ROC-AUC using an increasing number of top-ranked features according to Gl and mRMR ranking. $\mathbf{b}$ Gl ranking showing the top-ranked classification features of each feature-group, computed from manual annotations (green) and automatic segmentations (blue) 
Table 6 The ten top-ranked classification features according to Gini importance and minimum-redundancy-maximum-relevance

\begin{tabular}{|c|c|c|c|c|}
\hline \multirow[t]{2}{*}{ Rank } & \multicolumn{2}{|l|}{ Manual annotation } & \multicolumn{2}{|c|}{ Automatic segmentation } \\
\hline & Gini importance & Minimum-redundancy-maximum-relevance & Gini importance & Minimum-redundancy-maximum-relevance \\
\hline 1 & $\mathbf{f}_{\text {-tex-post/peak }}$ entropy & $\mathbf{f}_{\text {-tex-post/peak }}$ energy & $\mathbf{f}_{\text {l-kinetic }} T_{1 / 2}$ & $\mathbf{f}_{\text {-tex-post/peak }}$ energy \\
\hline 2 & $\mathbf{f}_{\text {-tex-post/peak }}$ energy & $\mathbf{f}_{\mathrm{l}-\delta c k c} \# 11$ & $\mathbf{f}_{\text {-tex-post/peak }}$ energy & $\mathbf{f}_{\mathrm{l}-\delta c k c} \# 10$ \\
\hline 3 & $\mathbf{f}_{\text {-kinetic }} T_{1 / 2}$ & $\mathbf{f}_{\mathrm{l}-\delta \mathrm{ckc}} \# 8$ & $\mathbf{f}_{\text {-kinetic }}$ MDER & $\mathbf{f}_{\text {-tex-post/pre sum average }}$ \\
\hline 4 & $\mathbf{f}_{\text {l-ckc }} \# 11$ & $\mathbf{f}_{\text {l-kinetic }}$ MDER & $\mathbf{f}_{\text {l-adc }}$ & $\mathbf{f}_{\text {-tex-post/pre }}$ homogeneity \\
\hline 5 & $\mathbf{f}_{\mathrm{l}-\mathrm{ckc}} \# 9$ & $\mathbf{f}_{\text {-tex-post/pre }}$ homogeneity & $\mathbf{f}_{1-\delta c k c} \# 18$ & $\mathbf{f}_{\mathrm{l}-\delta \mathrm{ckc}} \# 20$ \\
\hline 6 & $\mathbf{f}_{\text {-tex-post }}$ information measure 1 & $\mathbf{f}_{\text {-tex-post/peak correlation }}$ & $\mathbf{f}_{\mathrm{l}-\mathrm{ckc}} \# 11$ & $\mathbf{f}_{\text {-tex-post/peak }}$ information measure 2 \\
\hline 7 & $\mathbf{f}_{1-\delta c k c} \# 14$ & $\mathbf{f}_{\text {-tex-post/peak }}$ entropy & $\mathbf{f}_{\mathrm{l}-\delta c k c} \# 21$ & $\mathbf{f}_{\mathrm{l}-\delta \mathrm{ckc}} \# 21$ \\
\hline 8 & $\mathbf{f}_{\text {-tex-post }}$ information measure 2 & $\mathbf{f}_{\text {l-kinetic }} T_{1 / 2}$ & $\mathbf{f}_{1-\delta c k c} \# 14$ & $\mathbf{f}_{\text {I-kinetic }} \beta$ \\
\hline 9 & $\mathbf{f}_{1-\delta c k c} \# 11$ & $\mathbf{f}_{\text {-tex-post/pre }} \mathrm{dva}$ & $\mathbf{f}_{\text {-tex-post/pre }} \mathrm{dva}$ & $\mathbf{f}_{\text {-tex-peak/pre }}$ homogeneity \\
\hline 10 & $\mathbf{f}_{\text {-tex-peak }}$ entropy & $\mathbf{f}_{\text {-tex-post/pre }}$ energy & $\mathbf{f}_{\mathrm{l}-\delta \mathrm{ckc}} \# 10$ & $\mathbf{f}_{\text {-tex-post }}$ sum variance \\
\hline
\end{tabular}

dva difference variance, MDER maximum derivative of kinetic regression function

\section{Discussion}

We present a novel data-driven combined breast lesion segmentation and classification system for mpI data with combined ${ }^{18}$ F-FDG-PET/MRI. This system automatically detects and segments potentially cancerous regions and classifies lesions as benign or malignant. Our results showed that automatic lesion segmentation was accurate and improved with information from all modalities, but even a small number of features were sufficient to achieve the reported maximum accuracy. On the other hand, our results showed that lesion classification largely drew on information from DCE-MRI, without benefitting from information from other modalities and parameters. The results are consistent with previous findings but add insights into the feasibility of a completely automated lesion segmentation and of classification from $\mathrm{mpI}$ data. The results were obtained by quantifying the information captured across multimodal mpI data and features, enabling the assessment of imaging protocols in this context.

Using combined mpI based on DCE-MRI, DWI, and ${ }^{18}$ F-FDG-PET in a CADe or CADx system is a novel promising approach for improving diagnostic accuracy [26]. Previously, CADe and CADx systems have been proposed for digital mammography to increase the rather moderate sensitivity [27] and to help in classifying lesions as benign or malignant [28]. Semi-automatic methods have been proposed for classifying each pixel as cancerous or non-cancerous using fuzzy c-means clustering [29] or Markov random field-based clustering of the time-series [30]. Moreover, methods designed to outline lesions using the active contour framework (i.e., autonomously and adaptive search of object contours based on image features and user interaction) have also been presented [31, 32]. Automatic segmentation methods, which may also be seen as CADe systems, have been proposed using machine-learning approaches based on intensity and textural features (co-occurrence, run-length) [20, 33-35]. Recently, an automated localisation of breast cancer lesions based on DCE-MRI was proposed by Gubern-Mérida et al. [36]. Multimodal approaches combining several modalities have been reported for PET/CT breast images: Han et al. [37] segmented lesions by applying a graph-based Markov random field method on a combined PET/CT image, taking advantage from both the high spatial resolution of $\mathrm{CT}$ and the functional information of PET. Lastly, several CADx methods that classify breast lesions as benign or malignant by exploring the DCE-MRI data have been proposed using morphology [38], lesion texture [39], contrast enhancement $[16,40]$, a combination of morphology and contrast enhancement [41], or a combination of morphology and texture [19, 21, 31, 42, 43]. State-of-the-art DCE-MRI CADx methods have been reported using various performance metrics, different datasets (e.g., malignant cases only), and differing aims (i.e., segmentation versus detection).

Using our system, we detected all malignant cases and missed two benign lesions. Detected lesions were classified as malignant with a sensitivity of $95 \%$. Using texture features, Woods et al. [20] and Yao et al. [35] previously reported an ROC-AUC of 0.999 and 0.984, respectively. However, Woods et al. performed the evaluation on the same subjects as used in training, and both these studies were conducted in a small set of malignant lesions only. Twellmann et al. [33] reported a ROC-AUC of 0.99 for lesion detection using LOOCV and DCE-MRI information. Vignati et al. [34] reported the performance of a fully automated system as a detection rate of 0.89 and a sensitivity of 0.98 at four false-positive cases per breast. In their study, the performance measure did not include false-positive areas. Gubern-Mérida et al. [36] used an automated method and achieved a sensitivity of $89 \%$ at four false-positive per normal case. As normal cases, 
they included patients with a BI-RADS rating of 1 or 2 , who were healthy subjects with benign findings.

For the task of automatic lesion segmentation, our study showed that $\mathrm{mpI}$ is beneficial, as evidenced by the increase of the DSC from 0.584 to 0.665. The high ranking of DWI features in both GI and mRMR feature selection models indicates that the addition DWI to DCE-MRI is especially beneficial in segmentation. We also found that lesion segmentation benefitted from the addition of PET, although the benefit was to a lesser extent than that of DWI. When both DWI and PET were added, the DSC was further improved; thus, our results suggest that PET has a complementary relationship with DWI. Interestingly, features describing the change of contrast between time-steps $\left(\mathbf{f}_{\delta \mathrm{dce}}\right.$ and $\left.\mathbf{f}_{\delta \mathrm{mri}}\right)$ received a good ranking in the mRMR feature selection model overall but a low ranking in the GI feature selection model. A likely reason is that while they contribute less information than the higher-ranked GI features, their contribution is orthogonal to the higher-ranked features. In our study, mRMR as a feature selection model provided slightly better results than GI. The moderate mean DSC score for lesion segmentation results from several reasons. First, the two undetected benign lesions exhibited very low contrast enhancement with a DSC of 0 , leading to a drop in the mean value. However, we kept these two benign cases in the dataset to evaluate whether additional parameters may allow the system to segment these challenging cases, which was not the case as reported. Second, additional areas of contrast uptake, such as vessels and enhancing parenchymal tissues, resulted in an increased false-positive rate. While DWI and ${ }^{18}$ F-FDG-PET image modalities increased automatic segmentation accuracy, mainly by reducing the false-positive cases, lesions with low contrast uptake could not be detected automatically. As good segmentation is important for the accurate classification of a lesion, we aim to improve the segmentation performance, e.g., by introducing heuristics that filter false-positive cases in a post-processing step in a future study, as proposed for instance by Vignati et al. [34] and Gubern-Mérida et al. [36] where morphologic and kinetic descriptors were used in a second step.

In our study, a high accuracy in lesion classification was achieved for both expert and automatic segmentation. However, the highest accuracy was achieved with manual segmentation and mRMR feature selection from DCE imaging data. Top-ranked features largely overlapped between GI and mRMR feature selection models; the exception was that $\mathbf{f}_{\text {l-adc }}$ was ranked highly by the GI feature selection model following only automatic segmentation. While the addition of DWI and ${ }^{18}$ F-FDG-PET to DCE-MRI was beneficial overall for lesion segmentation, lesion classification only improved slightly with these two modalities for GI feature selection following manual segmentation. Lesion classification for mRMR features selection was best without these two modalities. $\mathbf{f}_{\text {l-pet }}$ was lowly ranked, consistent with recent findings by Magometschnigg et al. [44] that indicate that quantitative ${ }^{18} \mathrm{~F}$-FDG-PET values are not helpful for breast cancer classification. On the other hand, the kinetic feature $\mathbf{f}_{1 \text {-kinetic }}$ received a high GI as well as high mRMR ranking. Textural features were top-ranked,

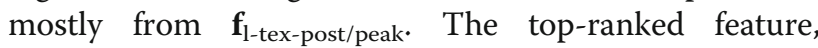
GLCM energy, measures the uniformity of lesion texture, reflecting the uniformity of contrast-enhancement within the lesion during a later stage. The morphologic feature $\mathbf{f}_{1 \text {-morph }}$ scored very low, although they are an integral part of the BI- RADS ${ }^{\otimes}$ lexicon for lesion classification, being discriminative features for clinical diagnosis, as shown by Pinker-Domenig et al. [45]. This suggests that binary segmentation and shape descriptors are not precise enough to describe the shape and margin of the lesion and feature extraction from a soft-margin around the hard segmentation border (e.g., textural features) may better capture the BI-RADS margin descriptors (circumscribed, non-circumscribed, irregular, spiculated). Alternatively, digital mammography or digital breast tomosynthesis may be used as an additional higher resolution modality to assess the morphology of the lesion more accurately. To summarise, mRMR slightly outperformed GI as a feature selection method for breast lesion classification. Novel DCE-MRI features that describe the kinetics and spatio-temporal texture of the contrast uptake were highly predictive for the classification of benign and malignant lesions, whereas DWI and PET did not provide additional information. Whereas we used data from separate MRI and PET/CT scanners, the methods, results, and findings can be directly transferred to images obtained at combined PET/MRI scanners, as the CT information was used for alignment only and was not part of the decision models.

One limitation of the study is that only subjects with suspicious findings on mammography or breast ultrasonography were included. As a consequence, an assessment of false-positive cases in healthy subjects was not possible. However, the majority of tissue in the breast consists of healthy tissue, on which the classifier was trained, and was classified as healthy tissue in our study. A second limitation is the small number of subjects. Even though cross-validation allowed us to estimate the generalisation of the model to some degree, statistical significance can only be obtained from a larger cohort. Thus, we aim to confirm our preliminary findings on a larger number of patients in a future study.

In conclusion, we used an entirely data-driven approach in combination with the assessment of the contribution of individual imaging parameters to provide a means for 
in-depth understanding of the multivariate information, where redundancies and relationships between imaging data are not obvious. This is essential for further clinical exploitation of imaging parameters. It enables designing of feasible imaging paradigms constructed from a possibly reduced subset of acquisition sequences. Furthermore, in the context of disease mechanisms, the data-driven model could serve as a means for hypothesis generation.

\section{Additional file}

Additional file 1: Figure S1. Illustration of the influence of logistic model parameters on curve, and the model fitted to a CKC. From left to right: a defines the asymmetry of the logistic model, $\tau$ the steepness of the curve and $k$ influences the terminal slope. The regression curve fitted to a given CKC for a malignant (blue) and a benign lesion (green). Figure S2. Boxplot of automatic segmentation performance in terms of Dice similarity coefficient (DSC). DWI, diffusion-weighted imaging; GI, Gini Importance; mRMR, minimum-Redundancy-Maximum-Relevance; PET, positron emission tomography; w/o, without. (DOCX $219 \mathrm{~kb}$ )

\section{Abbreviations \\ ${ }^{18} \mathrm{~F}$-FDG: ${ }^{18}$ F-fluorodeoxyglucose; ADC: Apparent diffusion coefficient; Bl- RADS: Breast Imaging-Reporting and Data System; CADe: Computer-aided detection; CADx: Computer-aided diagnosis; CT: Computed tomography; DCE-MRI: Contrast-enhanced magnetic resonance imaging; DSC: Dice similarity coefficient; DWI: Diffusion-weighted imaging; FLASH: Fast low angle shot; GI: Gini importance; GLCM: Grey-level co-occurrence matrix; LOOCV: Leave-one-out cross-validation; mpl: Multiparametric imaging; mRMR: Minimum-redundancy-maximum-relevance; PET: Positron emission tomography; RF: Random forest; ROC: Receiver operating characteristic; VIBE: Volumetric interpolated breath-hold-examination}

\section{Availability of data and materials}

The datasets used and/or analysed during the current study are available from the corresponding author on reasonable request.

\section{Funding}

This work was supported by the Austrian Science Fund FWF (12714-B31); the Austrian Federal Ministry of Economy, Family and Youth, the National Foundation for Research, Technology and Development [OPTIMA]; the Austrian Nationalbank 'Jubiläumsfond' Projects Nr. 16219 and Nr. 13418; and the H2020 - Research and Innovation Framework Programme PHC-11-2015 [667211-2]. Katja Pinker was partially supported by the $\mathrm{NIH} / \mathrm{NCl}$ Cancer Center Support Grant P30 CA008748.

\section{Acknowledgements}

The authors acknowledge the support in manuscript writing and editing from Joanne Chin.

\section{Authors' contributions}

$\mathrm{KP}, T H H$, and $\mathrm{GL}$ contributed to the conception and design. KP, THH, W-DV, and $G L$ contributed to the development of the methodology. W-DV, KP, $T H H, H B, G G, W B, S G$, and PD contributed to the acquisition of data (acquired and managed patients, provided facilities, etc.). W-DV, ZB-H, KP, and GL contributed to the analysis and interpretation of data (e.g., statistical analysis, biostatistics, computational analysis). W-DV, KP, THH, HB, GG, WB, SG, ZB-H, $P D$, and $G L$ contributed to the writing, review, and/or revision of the manuscript. W-DV, KP, THH, and GL contributed to the administrative, technical, or material support (i.e., reporting or organising data, constructing databases). KP, THH, and GL contributed to the study supervision. W-DV, KP, $\mathrm{THH}, \mathrm{HB}, \mathrm{GG}, \mathrm{WB}, \mathrm{SG}, \mathrm{ZB}-\mathrm{H}, \mathrm{PD}$, and $\mathrm{GL}$ contributed to the final approval of the manuscript to be published.

\section{Ethics approval and consent to participate}

The institutional review board of the Medical University of Vienna approved this prospective, single-institution study (EK 510_2009) and retrospective data analysis and all patients gave written, informed consent.

\section{Consent for publication}

Not applicable.

\section{Competing interests}

The authors declare that they have no competing interests.

\section{Publisher's Note}

Springer Nature remains neutral with regard to jurisdictional claims in published maps and institutional affiliations.

\section{Author details}

${ }^{1}$ Computational Imaging Research Laboratory, Department of Biomedical Imaging and Image-guided Therapy, Medical University Vienna, Waehringer Guertel 18-20, 1090 Vienna, Austria. ${ }^{2}$ Division of Molecular and Gender Imaging, Department of Biomedical Imaging and Image-guided Therapy, Medical University Vienna, 1090 Vienna, Austria. ${ }^{3}$ Department of Radiology, Breast Imaging Service, Memorial Sloan Kettering Cancer Center, New York, NY 10065, USA. ${ }^{4}$ MR Center of Excellence, Department of Biomedical Imaging and Image-guided Therapy, Medical University Vienna, 1090 Vienna, Austria. ${ }^{5}$ Department of Pathology, Medical University Vienna, 1090 Vienna, Austria. ${ }^{6}$ Department of Surgery, Medical University Vienna, 1090 Vienna, Austria. ${ }^{7}$ Department of Radiologic Technology, Carinthia University of Applied Sciences, Klagenfurt, Austria.

Received: 22 October 2018 Accepted: 7 March 2019

Published online: 27 April 2019

References

1. Ferlay J, Shin HR, Bray F, Forman D, Mathers C, Parkin DM (2010) Estimates of worldwide burden of cancer in 2008: GLOBOCAN 2008. Int J Cancer 127 : 2893-2917

2. Baum M (1976) The curability of breast cancer. BMJ 1:439-442

3. Pinker K, Bogner W, Baltzer P et al (2014) Improved differentiation of benign and malignant breast tumors with multiparametric 18fluorodeoxyglucose positron emission tomography magnetic resonance imaging: a feasibility study. Clin Cancer Res 20:3540-3549

4. Ayer T, Ayvaci MU, Liu ZX, Alagoz O, Burnside ES (2010) Computer-aided diagnostic models in breast cancer screening. Imaging Med 2:313-323

5. Woods BJ (2008) Computer-aided detection of malignant lesions in dynamic contrast enhanced MRI breast and prostate cancer datasets. Dissertation, Ohio State University Available via http://rave.ohiolink.edu/ etdc/view?acc_num=osu1218155270

6. Doi K (2007) Computer-aided diagnosis in medical imaging: historical review, current status and future potential. Comput Med Imaging Graph 31: $198-211$

7. Vyborny CJ, Giger ML, Nishikawa RM (2000) Computer-aided detection and diagnosis of breast cancer. Radiol Clin North Am 38:725-740

8. Morris E, Comstock C, Lee C, Lehman C, Ikeda D, Newstead G (2013) ACR BIRADS $^{\circledR}$ magnetic resonance imaging. ACR BI-RADS ${ }^{\circledR}$ Atlas, Breast Imaging Reporting and Data System Reston. American College of Radiology, VA, USA

9. Stoutjesdijk MJ, Fütterer JJ, Boetes C, van Die LE, Jager G, Barentsz JO (2005) Variability in the description of morphologic and contrast enhancement characteristics of breast lesions on magnetic resonance imaging. Investig Radiol 40:355-362

10. Breiman $L$ (2001) Random forests. Mach Learn 45:5-32

11. Pinker K, Grabner G, Bogner W et al (2009) A combined high temporal and high spatial resolution 3 Tesla MR imaging protocol for the assessment of breast lesions: initial results. Invest Radiol 44:553-558

12. Bogner W, Pinker-Domenig K, Bickel H et al (2012) Readout-segmented echo-planar imaging improves the diagnostic performance of diffusionweighted MR breast examinations at 3.0 T. Radiology 263:64-76

13. Avants BB, Tustison NJ, Song G, Cook PA, Klein A, Gee JC (2011) A reproducible evaluation of ANTs similarity metric performance in brain image registration. Neuroimage 54:2033-2044 
14. Somer EJ, Benatar NA, O'Doherty MJ, Smith MA, Marsden PK (2007) Use of the CT component of PET-CT to improve PET-MR registration: demonstration in soft-tissue sarcoma. Phys Med Biol 52:6991-7006

15. Adams R, Bischof $L$ (1994) Seeded region growing. IEEE Trans Pattern Anal Mach Intell 16:641-647

16. Chen W, Giger ML, Bick U, Newstead GM (2006) Automatic identification and classification of characteristic kinetic curves of breast lesions on DCEMRI. Med Phys 33:2878-2887

17. Haralick RM (1979) Statistical and structural approaches to texture. Proc IEEE 67:786-804

18. Chen W, Giger ML, Li H, Bick U, Newstead GM (2007) Volumetric texture analysis of breast lesions on contrast-enhanced magnetic resonance images. Magn Reson Med 58:562-571

19. Agner SC, Soman S, Libfeld E et al (2011) Textural kinetics: a novel dynamic contrast-enhanced (DCE)-MRI feature for breast lesion classification. J Digit Imaging 24:446-463

20. Woods BJ, Clymer BD, Kurc T et al (2007) Malignant-lesion segmentation using 4D co-occurrence texture analysis applied to dynamic contrastenhanced magnetic resonance breast image data. J Magn Reson Imaging 25:495-501

21. Nie K, Chen JH, Yu HJ, Chu Y, Nalcioglu O, Su MY (2008) Quantitative analysis of lesion morphology and texture features for diagnostic prediction in breast MRI. Acad Radiol 15:1513-1525

22. Gilhuijs KG, Giger ML, Bick U (1998) Computerized analysis of breast lesions in three dimensions using dynamic magnetic-resonance imaging. Med Phys 25:1647-1654

23. Dice LR (1945) Measures of the amount of ecologic association between species. Ecology 26:297-302

24. Peng H, Long F, Ding C (2005) Feature selection based on mutual information: criteria of max-dependency, max-relevance, and minredundancy. IEEE Trans Pattern Anal Mach Intell 27:1226-1238

25. Menze BH, Kelm BM, Masuch $\mathrm{R}$ et al (2009) A comparison of random forest and its Gini importance with standard chemometric methods for the feature selection and classification of spectral data. BMC Bioinformatics 10:213

26. Pinker K, Bogner W, Baltzer P et al (2014) Improved diagnostic accuracy with multiparametric magnetic resonance imaging of the breast using dynamic contrast-enhanced magnetic resonance imaging, diffusion-weighted imaging, and 3-dimensional proton magnetic resonance spectroscopic imaging. Invest Radiol 49:421-430

27. Oliver A, Freixenet J, Marti J et al (2010) A review of automatic mass detection and segmentation in mammographic images. Med Image Anal 14:87-110

28. Elter M, Horsch A (2009) CADx of mammographic masses and clustered microcalcifications: a review. Med Phys 36:2052-2068

29. Chen W, Giger ML, Bick U (2006) A fuzzy c-means (FCM)-based approach for computerized segmentation of breast lesions in dynamic contrast-enhanced MR images. Acad Radiol 13:63-72

30. Wu Q, Salganicoff M, Krishnan A, Fussell DS, Markey MK (2006) Interactive lesion segmentation on dynamic contrast enhanced breast MRI using a Markov model. Proc SPIE 6144:61444M-1-61444M-8. https://doi.org/10.1117/ 12.654308

31. Zheng Y, Englander S, Baloch S et al (2009) STEP: spatiotemporal enhancement pattern for MR-based breast tumor diagnosis. Med Phys 36: 3192-3204

32. Agner SC, Xu J, Madabhushi A (2013) Spectral embedding based active contour (SEAC) for lesion segmentation on breast dynamic contrast enhanced magnetic resonance imaging. Med Phys 40:032305

33. Twellmann T, Lichte O, Nattkemper TW (2005) An adaptive tissue characterization network for model-free visualization of dynamic contrastenhanced magnetic resonance image data. IEEE Trans Med Imaging 24: 1256-1266

34. Vignati A, Giannini V, De Luca M et al (2011) Performance of a fully automatic lesion detection system for breast DCE-MRI. J Magn Reson Imaging 34:1341-1351

35. Yao J, Chen J, Chow C (2009) Breast tumor analysis in dynamic contrastenhanced MRI using texture features and wavelet transform. IEEE J Sel Top Signal Process 3:94-100

36. Gubern-Mérida A, Martí R, Melendez J et al (2015) Automated localization of breast cancer in DCE-MRI. Med Image Anal 20:265-274

37. Han D, Bayouth J, Song Q et al (2011) Globally optimal tumor segmentation in PET-CT images: a graph-based co-segmentation method. In: Székely G,
Hahn HK (eds) Information Processing in Medical Imaging. IPMI 2011. Lecture Notes in Computer Science, vol 6801. Springer, Berlin, Heidelberg, pp 245-256

38. Meinel LA, Stolpen AH, Berbaum KS, Fajardo LL, Reinhardt JM (2007) Breast MRI lesion classification: improved performance of human readers with a backpropagation neural network computer-aided diagnosis (CAD) system. J Magn Reson Imaging 25:89-95

39. Gibbs P, Turnbull LW (2003) Textural analysis of contrast-enhanced MR images of the breast. Magn Reson Med 50:92-98

40. Levman J, Leung T, Causer P, Plewes D, Martel AL (2008) Classification of dynamic contrast-enhanced magnetic resonance breast lesions by support vector machines. IEEE Trans Med Imaging 27:688-696

41. Szabó BK, Wiberg MK, Boné B, Aspelin P (2004) Application of artificial neural networks to the analysis of dynamic MR imaging features of the breast. Eur Radiol 14:1217-1225

42. McLaren CE, Chen WP, Nie K, Su MY (2009) Prediction of malignant breast lesions from MRI features: a comparison of artificial neural network and logistic regression techniques. Acad Radiol 16:842-851

43. Chen W, Giger ML, Newstead GM et al (2010) Computerized assessment of breast lesion malignancy using DCE-MRI robustness study on two independent clinical datasets from two manufacturers. Acad Radiol 17:822-829

44. Magometschnigg HF, Baltzer PA, Fueger B et al (2015) Diagnostic accuracy of 18F-FDG PET/CT compared with that of contrast-enhanced MRI of the breast at 3 T. Eur J Nucl Med Mol Imaging 42:1656-1665

45. Pinker-Domenig K, Bogner W, Gruber S et al (2012) High resolution MRI of the breast at $3 \mathrm{~T}$ : which BI-RADS ${ }^{\oplus}$ descriptors are most strongly associated with the diagnosis of breast cancer. Eur Radiol 22:322-330

\section{Submit your manuscript to a SpringerOpen ${ }^{\circ}$ journal and benefit from:}

- Convenient online submission

- Rigorous peer review

- Open access: articles freely available online

- High visibility within the field

- Retaining the copyright to your article

Submit your next manuscript at $>$ springeropen.com 